\title{
Reflets
}

Revue ontaroise d'intervention sociale et communautaire

\section{Vers des services attirants, accessibles et pertinents pour les personnes âgées immigrantes francophones?}

\section{Mathilde Nduwimana et Alice Home}

Volume 1, numéro 1, printemps 1995

Des pratiques à notre image : défis et ressources

URI : https://id.erudit.org/iderudit/026056ar

DOI : https://doi.org/10.7202/026056ar

Aller au sommaire du numéro

Éditeur(s)

Reflets : Revue ontaroise d'intervention sociale et communautaire

ISSN

1203-4576 (imprimé)

1712-8498 (numérique)

Découvrir la revue

Citer cet article

Nduwimana, M. \& Home, A. (1995). Vers des services attirants, accessibles et pertinents pour les personnes âgées immigrantes francophones? Reflets, 1(1), 92-121. https://doi.org/10.7202/026056ar

\section{Résumé de l'article}

Cet article décrit et analyse les besoins et préoccupations des personnes âgées autonomes appartenant aux groupes ethniques francophones et résidant dans Ottawa-Carleton. L'objectif principal est d'explorer les raisons pour lesquelles elles utilisent peu les services de soutien communautaires pour les personnes âgées francophones. Nous avons effectué des entrevues semi-structurées auprès de quatre représentantes et de six personnes âgées des communautés ethno-culturelles francophones, et nous avons effectué une analyse de contenu par thème. Les répondantes ont souligné les besoins de contacts sociaux, d'apprentissage du français et/ou de l'anglais, d'accès aux moyens financiers adéquats et d'information sur les ressources disponibles. Des obstacles liés au contexte socio-écono-politique dans la société d'accueil entravent la satisfaction de ces besoins. La levée de certaines barrières concrètes, la modification des politiques sociales et des pratiques d'intervention faciliteraient l'accès aux services par les personnes âgées des groupes ethniques francophones et augmenteraient leurs options.
Tous droits réservés (C Reflets : Revue ontaroise d'intervention sociale et communautaire, 1995
Ce document est protégé par la loi sur le droit d'auteur. L'utilisation des services d'Érudit (y compris la reproduction) est assujettie à sa politique d'utilisation que vous pouvez consulter en ligne. 


\section{Vers des services attirants, accessibles et pertinents pour les personnes âgées immigrantes francophones?}

Cet article décrit et analyse les besoins et préoccupations des personnes âgées autonomes appartenant aux groupes ethniques francophones et résidant dans Ottawa-Carleton. L'objectif principal est d'explorer les raisons pour lesquelles elles utilisent peu les services de soutien communautaires pour les personnes âgées francophones. Nous avons effectué des entrevues semi-structurées auprès de quatre représentantes et de six personnes âgées des communautés ethno-culturelles francophones, et nous avons effectué une analyse de contenu par thème. Les répondantes ont souligné les besoins de contacts sociaux, d'apprentissage du français et /ou de l'anglais, d'accès aux moyens financiers adéquats et d'information sur les ressources disponibles. Des obstacles liés au contexte socio-écono-politique dans la société d'accueil entravent la satisfaction de ces besoins. La levée de certaines barrières concrètes, la modification des politiques sociales et des pratiques d'intervention faciliteraient l'accès aux services par les personnes âgées des groupes ethniques francophones et augmenteraient leurs options.

\section{Mathilde Nduwimana et Alice Home \\ École de service social, Université d'Ottawa}

\section{Introduction}

Nous assistons actuellement à une augmentation du nombre de personnes âgées des groupes francophones en Ontario comme ailleurs au Canada, surtout à cause du vieillissement de la population canadienne et de l'augmentation du nombre d'immigrantes et immigrants provenant des pays du Tiers-Monde (Labelle, 1988; 
"...les personnes âgées des groupes ethniques francophones utilisent moins les services et participent moins aux activités organisées que les personnes âgées francophones de souche.»
Beaulieu et al., 1994). Les politiques de regroupement familial et d'acceptation des personnes retraitées contribuent à accentuer la diversité socio-culturelle de la population âgée. Ottawa-Carleton, on a estimé à plus ou moins 6000 (Services aux aînés d'Ottawa Ouest, 1988), le nombre de personnes âgées provenant des communautés ethno-culturelles francophones. Les principales composantes de ces communautés ethno-culturelles francophones sont les Africains, les Haïtiens, les Libanais et lesVietnamiens (Beaulieu et al., 1994:18).

L'étude statistique sur le vieillissement de 1991 révèle qu'environ $15 \%$ de la population âgée de 65 ans et plus d'OttawaCarleton parle le français à la maison, $8 \%$ utilise une langue non officielle, alors que 2,5\% parle plus d'une langue. Dans cette région, on retrouve environ 1300 personnes âgées des groupes ethniques francophones qui s'expriment en français (Racine, 1994). La plupart de ces personnes préfèrent être servies en français plutôt qu'en anglais.

Par ailleurs, la plupart des organismes de soutien communautaires francophones de la région signalent que les personnes âgées des groupes ethniques francophones utilisent moins les services et participent moins aux activités organisées que les personnes âgées francophones de souche. Elles participent aussi très peu aux comités d'organismes communautaires s'occupant des affaires du troisième âge. Plusieurs obstacles peuvent réduire l'accessibilité des minorités ethniques aux services sociaux et de santé, en particulier chez les personnes âgées dont les besoins spécifiques méritent une attention particulière (Bergin, 1988). Alors que la plupart des personnes âgées vivent des pertes, celles qui sont immigrantes peuvent être soudainement coupées de leurs réseaux social et familial, en plus de vivre la perte du statut, du prestige social et des biens matériels (Eyre, 1992). Confrontées à des coutumes et à des valeurs culturelles nouvelles, surmontant difficilement les obstacles à leur bien-être, la plupart d'entre elles ont de la difficulté à trouver les services dont elles ont réellement besoin.

Même si les services sociaux deviennent plus sensibles à la nécessité d'adapter leurs services aux besoins des groupes ethniques, (Herberg, 1993; Jacob et Bertot, 1991), il existe peu 
Reflets

de recherches pertinentes pour appuyer cette démarche. Parmi les rares études canadiennes sur les personnes âgées des groupes ethniques, notons celle menée par Ujimoto (1987), qui a examiné l'organisation des activités, les facteurs culturels et le bienêtre des personnes âgées canadiennes d'origine japonaise, et celle de Disman (1987), qui a exploré l'expérience vécue par une personne âgée immigrante au Canada. Il n'existe aucune recherche sur les besoins des personnes âgées des groupes ethniques francophones relativement à l'utilisation des services dans OttawaCarleton. Notre étude vise alors à mieux comprendre les conditions de vie des personnes âgées des groupes ethniques francophones et les approches susceptibles de les rejoindre.

Notre recherche-intervention s'est réalisée entre janvier et avril 1994, dans le cadre d'un stage de maitrise en service social au Conseil sur le vieillissement d'Ottawa-Carleton. Nos objectifs étaient d'identifier les besoins et préoccupations des personnes âgées des groupes ethniques francophones d'Ottawa-Carleton, et d'explorer les raisons pour lesquelles elles utilisent moins les services de soutien communautaires francophones pour les aînées et aînés. L'étude visait à recueillir des données dont les organismes pourraient tenir compte dans la conceptualisation, la planification et la coordination des services. Elle cherchait de plus à développer des stratégies pour encourager ces personnes à participer aux activités socio-culturelles et aux comités s'occupant des affaires les concernant, afin de revendiquer leurs droits et de contribuer aux prises de décisions.

\section{Être personne âgée de groupe ethnique francophone}

Pour la plupart des personnes âgées immigrantes, la vieillesse se définit et se vit différemment des aînées et aînés francophones de souche. Pour celles en provenance des pays du Tiers-Monde, la vieillesse incarne la connaissance et l'expérience accumulées au cours de la vie. La tradition orale et coutumière leur attribue les rôles de faciliter le lien entre les générations et de maintenir 
"Les immigrantes ou immigrants aînés sont souvent sujets à une double oppression, liée à la fois à leur condition d'ainé et de membres d'une minorité visible.» intacte la mémoire collective des coutumes et traditions. Dans ces sociétés, la personne âgée doit remplir les rôles sociaux préétablis en fonction des valeurs culturelles dominantes, et ne peut pas choisir de vivre autrement sous peine d'exclusion (Laforest, 1989:13; Minois, 1987). Par contre, les membres de ces sociétés offrent à la personne âgée un respect rarement présent dans les sociétés occidentales.

En immigrant, les personnes âgées des groupes ethniques rencontrent des modes de vie et des coutumes radicalement différents de ceux qu'elles ont connus dans leurs pays d'origine. L'accueil réservé aux immigrantes et immigrants n'est souvent pas favorable à une bonne adaptation et intégration (Santé Bienêtre Canada et Multiculturalisme, 1988; Bertot et Jacob, 1991). Plusieurs témoignages font état du fait qu'elles sont toujours considérées comme des étrangères, même lorsqu'elles ont passé plusieurs années au Canada (Baukje et Nason-Clark, 1989). Les immigrantes ou immigrants ainés sont souvent sujets à une double oppression, liée à la fois à leur condition d'aîné et de membres d'une minorité visible. Les femmes âgées de minorités visibles vivent une triple oppression du fait additionnel d'être âgées dans une société où la jeunesse est plus valorisée. Ces éléments de discrimination sont fortement ancrés au sein des institutions de la société d'accueil (Naidoo et Edwards, 1990).

Les politiques canadiennes de multiculturalisme facilitent la sauvegarde des cultures des pays d'origine. Cependant, le fait de tenir à sa culture d'origine peut gêner l'adaptation et l'intégration dans la société d'accueil. Certains éléments périphériques de la vie sociale des adultes contribuent à une acculturation aux normes de la société globale, même si le «noyau culturel dur» demeure souvent intact et continue de refléter la culture d'origine (Abou, 1988:5). Cette acculturation se fait suite à l'intégration fonctionnelle dans la société, par l'apprentissage de l'une ou des deux langues de la société d'accueil ou par le travail. Pour les personnes âgées qui ont moins besoin de s'accommoder aux exigences fonctionnelles (telles que l'emploi) de la société d'accueil, le processus d'intégration est compliqué par le fait qu'elles peuvent plus facilement rester en retrait des activités quotidiennes. Il 
Reflets

"Les personnes âgées des groupes ethniques ont souvent un niveau de formation moins élevé que celles nées au Canada.»

"La situation financière de la plupart des personnes âgées des groupes ethniques demeure souvent précaire.» devient ainsi difficile de tisser de nouveaux liens sociaux pour remplacer ceux perdus suite à l'immigration (Duval, 1991) et de faciliter l'intégration en apprenant les langues officielles du Canada par des échanges interpersonnels.

Les personnes âgées des groupes ethniques ont souvent un niveau de formation moins élevé que celles nées au Canada. La majorité de ces personnes seraient même analphabètes en termes d'écriture et de lecture du français ou de l'anglais, tout en ayant de la difficulté à s'exprimer dans les deux langues officielles du Canada. Cela peut nuire à l'obtention d'informations au sujet des services disponibles, réduisant aussi leur accès au travail, à l'éducation, aux services sociaux et à d'autres institutions (Donald et Barresi, 1987:30).

La situation financière de la plupart des personnes âgées des groupes ethniques demeure souvent précaire. On constate que les immigrantes et immigrants occupent souvent des emplois subalternes, mal protégés et mal rémunérés, qui n'offrent aucune possibilité de promotion (Demers, 1987; Labelle, 1987). On peut alors s'imaginer ce que peut représenter le poids d'avoir un parent âgé à supporter pendant dix ans, comme l'exigent les politiques sociales régissant le parrainage d'un parent. Les personnes âgées immigrantes ne peuvent en fait bénéficier d'aucune sorte de pension avant d'avoir vécu pendant dix années consécutives au Canada et d'être âgées de 65 ans (MacDonald et Wanner, 1990:114). Quant aux personnes âgées de groupes ethniques qui sont arrivées au Canada à l'âge adulte, on remarque que leur situation économique n'est pas non plus satisfaisante. Elles ne peuvent pas bénéficier de l'entièreté de la pension de la vieillesse, à moins d'avoir vécu au Canada pendant 40 années (McDonald et Wanner, 1990:114). Cela entraîne des répercussions plus marquées pour les personnes âgées de sexe féminin qui ont très peu d'opportunités d'intégrer le marché de l'emploi et d'y rester longtemps. Comme résultat, plusieurs personnes âgées des groupes ethniques passent des années sans aucune source de revenu, devenant ainsi totalement dépendantes de leurs enfants, avec beaucoup de risques de conflits entre les parents âgés et leurs enfants. 


\section{Utilisation des ressources par les personnes âgées francophones}

Les personnes âgées ont accès à diverses ressources formelles et informelles pour répondre à leurs besoins. Les ressources formelles telles que les hôpitaux et les services sociaux offrent des services sans frais. Parmi les ressources informelles, on retrouve les services organisés au sein des organismes communautaires et ceux offerts par les membres d'une communauté donnée. Les membres d'une communauté (famille, amis, voisins) se donnent mutuellement ce soutien social, affectif ou tangible pour faire face aux difficultés de la vie quotidienne. La plupart des personnes âgées des groupes ethniques ne disposent pas d'un réseau familial ou social sur lequel elles ou ils peuvent compter pour ce genre de soutien.

L'utilisation des ressources communautaires par les personnes âgées dépend de plusieurs facteurs. On en dénombre trois principales catégories, à savoir: les facteurs facilitants tels que la distance et les possibilités d'accès aux services, les facteurs prédisposants dans la demande et l'utilisation des services (entre autres l'âge, la race, l'éducation et la perception des services) ainsi que la nature et l'acuité des besoins. (Bertot et Jacob, 1991:63; Gelfand et Barresi, 1987 et Bibeau, 1987:7980; McNeely et Colen, 1983:246). La demande et l'utilisation des services dépendent aussi des revenus, de l'assurance sociale, des moyens de transport disponibles, de la disponibilité et de l'accessibilité des services (Gelfand et Barresi, 1987; Neysmith, 1985).

D'autres facteurs personnels et structurels peuvent entrer en jeu. Certaines des clientèles dites difficiles à rejoindre, peuvent être conscientes de leurs situations problématiques et ne pas demander des services, parce qu'elles perçoivent les situations qu'elles vivent comme étant naturelles. D'autres sont conscientes des problèmes et des services qu'elles peuvent utiliser, mais à cause d'expériences négatives avec certains services, deviennent réticentes à l'égard de tous les services (Breton, 1991). Le soutien informel, l'interface 
Reflets

entre celui-ci et le soutien formel, sont aussi des facteurs qui ont un impact sur l'utilisation des services par les aînées et aînés (Darveau-Fournier et Home, 1989).

\section{Utilisation des services par les personnes âgées des groupes ethniques}

"Lorsque l'assistance familiale est absente, ces personnes préfêrent

celle des membres de leurs communautés et/ou des organisations religieuses...»
En général, les personnes âgées des groupes ethniques n'aiment pas beaucoup utiliser des services socio-sanitaires, préférant recevoir l'assistance de leurs propres familles (Gelfand et Barresi, 1987:199; Guttmann, 1985). Lorsque l'assistance familiale est absente, ces personnes préferent celle des membres de leurs communautés et/ou des organisations religieuses, parce qu'elles ne connaissent pas les services disponibles et hésitent à faire appel à des ressources qui ne leur sont pas familières. Les valeurs culturelles de ces personnes, qui croient qu'une demande d'aide à des étrangers risquerait de diminuer l'estime de leur entourage à leur endroit, peuvent aussi être associées à cette façon de fonctionner (Legault et Lafrenière, 1992:45). Lorsqu'elles arrivent à solliciter l'assistance en dehors de ces réseaux et que leurs demandes ne sont pas satisfaites, les personnes ainées peuvent se sentir à la fois isolées socialement et culturellement. Gelfand et Barresi (1987:200) notent aussi que les membres des familles ethno-culturelles sont souvent réticents à placer leurs parents âgés dans des institutions, préférant souvent s'en occuper eux-mêmes, dans leur propre milieu. Cependant, les conditions de vie dans le pays d'accueil ne le permettent pas toujours et, de surcroît, ces personnes ont besoin de services que les communautés ethniques ne sont pas toujours en mesure d'offrir.

Les personnes âgées et leurs aidantes ou aidants naturels peuvent aussi avoir des difficultés à franchir les barrières inhérentes à l'organisation bureaucratique de services, tout comme ils peuvent se heurter à la fragmentation des services dont l'utilisation demande d'être assez persévérant et informé. L'emplacement des 
services, surtout leur éloignement par rapport au milieu de vie des personnes âgées, peut constituer un facteur additionnel entravant leur utilisation, à cause du temps et des coûts encourus pour s'y rendre (Breton, 1985; Legault, 1993).

Pendant l'hiver, la température et la crainte de tomber empêchent plusieurs personnes âgées de se déplacer. Les personnes âgées des groupes ethniques francophones peuvent aussi avoir des difficultés à s'affirmer pour obtenir les services, subséquemment au fait de ne pas être habituées à revendiquer, dû au respect de l'autorité dans la plupart de leurs pays d'origine (Legault et Lafrenière, 1992 et Breton, 1992).

\section{Méthodologie de l'étude}

Dans cette recherche exploratoire, nous avons utilisé une approche méthodologique qualitative pour effectuer des entretiens semidirigés auprès des représentantes et des personnes âgées des groupes ethniques francophones. Notre échantillonnage des représentantes ou représentants a été fait par choix délibéré, afin d'interroger les personnes occupant des postes clés qui connaissent bien le sujet à l'étude (Grinnell, 1988) et par contraste, pour identifier des associations ethno-culturelles les plus différentes possibles, afin de relever des similarités ou des divergences (Patton, 1982). Le recrutement a été fait en répertoriant les associations ethnoculturelles francophones d'Ottawa-Carleton, en contactant les responsables et ensuite les personnes représentantes identifiées.

Le recrutement des personnes âgées des groupes ethniques d'Ottawa-Carleton s'est effectué par la technique d'échantillonnage boule de neige, qui consiste à ajouter à un noyau d'individus tous ceux qui sont en relation avec eux (Mayer et Ouellet, 1991:390). Conscientes de la difficulté de rejoindre ces personnes âgées à cause de leur éparpillement géographique, nous avons opté pour la méthode d'information de bouche à oreille où les représentantes ou représentants des communautés ciblées ont 
Reflets

identifié les personnes âgées de leurs communautés qui désiraient participer à l'étude. Cette méthode est aussi compatible avec les valeurs culturelles de plusieurs communautés ethniques.

Nous avons élaboré deux guides d'entretien semi-structuré, constitués de questions touchant les thèmes de la recherche (Mayer et Ouellet, 1991:315). Le choix de cette méthode visait à faciliter la bonne compréhension des questions, vu que la plupart des personnes âgées concernées ont des difficultés à lire et à écrire le français, certaines d'entre elles ayant évolué dans une culture caractérisée par la tradition orale. Cela nous a permis d'augmenter le taux de réponses aux questions et d'améliorer la validité du contenu des informations obtenues (Deslauriers, 1991). Un premier guide d'entretien était destiné aux aînées et aînés des communautés ethno-culturelles francophones, alors que le deuxième a été préparé pour les représentantes des communautés ethnoculturelles francophones. Les guides ont été adaptés à chaque communauté, en fonction des coutumes et traditions touchant les manières d'entrer en contact avec les gens et de poser des questions sans les offenser.

Nous avons interviewé quatre représentantes des communautés ethno-culturelles et six personnes âgées concernées par l'étude. Les représentantes interviewées oeuvrent dans des associations de leurs communautés respectives et les entrevues se sont déroulées dans leurs bureaux de travail. Pour ce qui est des six personnes âgées, nous les avons rencontrées à leurs domiciles, afin d'assurer une atmosphère détendue et confortable. Nous n'avons pas enregistré ces entrevues parce que les personnes âgées, comme d'autres immigrants en général, n'aiment pas se faire enregistrer ni s'engager par écrit,de peur d'éventuelles dénonciations. Par contre, nous avons pris des notes pendant et après l'entrevue pour nous assurer de bien refléter ce que la personne avait exprimé.

Les entrevues ont été transcrites et codées en nous appuyant sur la technique de codage et de classification et d'analyse des données de Huberman et Miles (1991). Cette méthode permet de classifier des éléments d'un message afin de dégager les différentes caractéristiques, en vue d'en faire comprendre le sens. Nous avons enfin confronté les données ainsi classifiées à la théorie, en 
faisant ressortir les tendances générales et en soulignant les ressemblances et divergences entre les discours des différents acteurs.

\section{Difficultés et limites de la recherche}

Cette recherche-intervention a permis de constater à quel point la période d'apprivoisement du milieu interculturel et le recrutement de participantes ou participants n'est pas facile. Nous avons eu à nous adapter continuellement à chacune d'entre elles, en nous informant d'avance sur les coutumes et traditions des différentes communautés afin d'essayer de contourner les résistances à participer à la recherche. Nous sommes d'accord avec Legault et Fortin (1993) que l'apprivoisement réciproque oblige à franchir beaucoup d'étapes avant d'arriver à fixer les entretiens et occasionne des délais dans la recherche. Les barrières culturelles, en liaison avec la religion et les pratiques culturelles, ont aussi affecté le déroulement de notre recherche. Par exemple, nous n'avons pas pu recruter et effectuer des entrevues avec les personnes âgées de la communauté somalienne, puisqu'elle était en période de Ramadan. L'adaptation continuelle des guides d'entrevue afin de ne pas offenser les répondantes ou répondants par la façon de poser des questions a ralenti un processus qui aurait été moins compliqué dans une communauté culturellement homogène.

Des circonstances indépendantes de notre volonté ont fait que nous avons recruté uniquement des femmes représentantes des communautés et que cinq des six personnes âgées étaient de sexe féminin. Nous estimons qu'il faut être prudent avant de généraliser les données, compte tenu de la taille de l'échantillon et du fait que la recherche-intervention se soit déroulée dans une seule région. La recherche ayant aussi été limitée par l'impossibilité d'enregistrer les entrevues avec les personnes âgées, certains détails des informations partagées peuvent nous avoir échappé. Le fait d'être la seule chercheure peut aussi avoir introduit une certaine subjectivité au moment de l'interprétation et de l'analyse des données, mais le fait de disposer de deux sources 
Reflets

de données a permis de contourner ces limites en effectuant des comparaisons sur certaines questions selon un processus de triangulation de sources des données (Denzin, 1978). Nous estimons tout de même que les résultats de l'étude représentent bien les expériences des personnes interviewées, et que les méthodes utilisées étaient pertinentes étant donné les objectifs exploratoires de l'étude.

\section{Présentation et analyse des résultats}

L'analyse des propos des deux catégories de participantes vise d'abord à identifier les facteurs qui engendrent des besoins ainsi que les obstacles qui entravent leur satisfaction, et ensuite à cerner des approches ou des stratégies indispensables pour surmonter ces barrières. Les informations recueillies ont permis d'identifier sept types de besoins, que nous avons regroupés en deux grandes catégories, à savoir, les besoins de base à caractère concret et ceux à caractère socio-affectif. Notons que les besoins concrets doivent être satisfaits avant même que les besoins à caractère socio-affectif puissent prendre de l'importance aux yeux de l'individu (Mayer et Ouellet, 1991:64).

\section{Besoins tangibles ou concrets}

Toutes les personnes participant à la recherche ont indiqué que la plupart des personnes âgées des groupes ethniques, et particulièrement celles parrainées par leurs enfants expriment des besoins concrets. On se rappelle que ce sont les enfants qui doivent s'occuper de la satisfaction de ces besoins au cours des dix années suivant l'arrivée des parents, mais que les ressources nécessaires pour ce faire restent insuffisantes. La totalité des représentantes et la plupart des personnes âgées interrogées ont également évoqué le 
«...la plupart des

personnes âgées

interrogées ont

également évoqué le

besoin d'interprètes

linguistiques et

culturels pour faciliter

la communication...» besoin d'interprètes linguistiques et culturels pour faciliter la communication, ainsi que le besoin d'accompagnement dans différentes situations. Elles ont aussi mentionné la nécessité d'embaucher des intervenantes ou intervenants issus des communautés ethniques francophones pouvant servir d'intermédiaire entre elles et le personnel des organismes de soutien communautaires, ainsi que l'importance de services qui seraient sensibles à leurs valeurs culturelles.

Toujours au niveau des besoins tangibles, les moyens de transport ont été soulignés par la totalité des répondantes et répondants. Une des personnes âgées s'est exprimée en ces termes: «Nous avons un problème de déplacement. Le transport est difficile car nous n'avons pas les moyens personnels pour nous déplacer...". D'autres besoins de cette nature comprennent les vêtements adaptés aux différentes saisons du pays d'accueil: «...je voudrais avoir de l'argent pour les achats, comme pour acheter un bon chandail, des vêtements chauds pour l'hiver...». Des lieux de rencontres socio-culturels et des professionnels sensibles à la culture des bénéficiaires ont également fait partie des propos tenus par l'ensemble des répondants.

Trois des quatre représentantes ont indiqué que les aînées et aînés de leurs communautés avaient des besoins économiques importants. La majorité des personnes âgées des groupes ethniques ne peuvent pas toucher une pension convenable, car elles n'ont pas travaillé au Canada, ou alors parce qu'elles ont occupé des emplois mal rémunérés et non stables (Demers 1987; Labelle, 1987). Cette situation financière précaire constitue un obstacle important, qui freine la satisfaction des besoins ou qui limite les choix qu'ont ces personnes d'obtenir des services de soutien communautaires payants. Obligées de dépendre de leurs enfants, elles perdent leur estime de soi et se sentent dévalorisées, comme l'a expliqué une des répondantes:

Je m'adresse à mes enfants pour tous mes besoins, même pour l'argent, ils me donnent un tout petit peu seulement. Je n'aime pas demander des choses car il $y$ a beaucoup de dépenses, beaucoup de factures à payer... 
Reflets

"...les personnes

âgées... ont besoin

d'être informées sur les normes et codes de fonctionnement de la nouvelle collecti-

vité...»
Mes enfants aiment me faire plaisir, mais moi je n'aime pas demander trop, car j'ai travaillé dans mon pays, et j'aurais souhaité que ce soit moi qui offre à mes enfants.

Il faut toutefois noter qu'il y a des personnes âgées qui n'ont pas souligné l'importance des besoins financiers chez les aînées de leurs communautés. Elles peuvent être satisfaites du peu de moyens financiers disponibles actuellement, qui se compare avantageusement à leur situation financière dans leur pays d'origine. Comme l'exprime une personne âgée: «C'est déjà généreux ce que je reçois, je suis contente de ça, par rapport à ce que nous vivions là-bas...» Il peut aussi être question de désirabilité sociale reliée à la culture, alors que certaines personnes peuvent avoir honte d'étaler leurs besoins.

L'ensemble des représentantes et des personnes âgées ont souligné des besoins importants en matière d'informations. Elles ont indiqué que les personnes âgées nouvellement arrivées ont besoin d'être informées sur les normes et codes de fonctionnement de la nouvelle collectivité, afin d'éviter des frustrations et des embarras subséquents au fait de poser des gestes contraires à ceux acceptés par les membres de la société d'accueil. Plusieurs obstacles rendent l'information inaccessible. Parmi les plus importants, nous avons noté les barrières linguistiques et culturelles reliées à l'analphabétisme en terme de lecture ou d'écriture du français. Le problème se situe aussi au niveau des incompréhensions possibles par rapport à l'intonation des interlocuteurs, car certaines personnes âgées n'arrivent pas à comprendre le français parlé ici. Ces personnes hésitent alors à utiliser certains services, comme l'a exprimé une d'entre elles:

J'ai peur d'aller participer aux activités parce que je ne comprends pas les termes utilisés par les agents des services, je ne voudrais pas aller rester dans le coin sans participer effectivement. Il $y$ a des termes qui sont propres à chaque culture et chacun les comprend à sa manière, à sa façon... 
"...les pressions

d'adaptation... entraînent des conflits intrafamiliaux ainsi que des problèmes de santé mentale.»
Le fait que les organismes de soutien communautaires francophones utilisent, pour se faire connaitre, des dépliants, des articles dans des journaux francophones et des annonces dans les médias pose problème pour les personnes âgées analphabètes, qui utilisent peu ces sources d'informations. De plus, les différents modèles de transmission de l'information peuvent ne pas être familiers aux personnes âgées nouvellement arrivées au Canada, étant donné que la transmission de l'information se fait de bouche à oreille dans plusieurs pays d'origine.

Nos données suggèrent que les personnes âgées des communautés ethniques ont des besoins de santé qui leur sont particuliers. Il s'agit de problèmes de santé mentale engendrés par l'angoisse, l'isolement, le stress occasionné par le processus d'adaptation et d'intégration au sein de la collectivité d'accueil, les conflits de génération, la tristesse liée à la perte des réseaux de leurs pays d'origine. Ceci rejoint l'observation selon laquelle les pressions d'adaptation des immigrantes et immigrants entraînent des conflits intrafamiliaux ainsi que des problèmes de santé mentale (Bibeau et al., 1992:113). Nous suggérons que ces facteurs affectent non seulement la santé mentale de ces personnes, mais qu'ils ont aussi des effets sur leur santé physique. Certaines personnes âgées en sont conscientes, et le contenu de ces extraits d'entrevues le traduit bien: "Quand on est souvent triste et angoissé, on a mal à l'estomac à cause de l'anxiété...». Une autre a dit: «Je pense souvent à ma famille restée là-bas, vous savez il y a des problèmes, et j'ai beaucoup de maux de tête,...».

Se retrouvant dans l'impossibilité de se confier aux proches pour bénéficier de leurs conseils et de soutien, comme elles le faisaient dans leur pays d'origine, ces personnes ne savent pas comment gérer ces problèmes de santé. Plusieurs d'entre elles ignorent les ressources susceptibles de les aider, et les approches utilisées ne leur sont pas familières. En outre, les personnes âgées parrainées par les enfants sont particulièrement vulnérables, dans la mesure où les choix de solutions sont limités. Une des représentantes a dit: "Nos aînées et aînés ont besoin de savoir comment faire pour répondre aux besoins de santé non-couverts par l'OHIP, comme les soins dentaires ou une partie des soins de la diabète.» 
Reflets

\section{Besoins à caractère socio-affectif}

"On se lève le matin, on va travailler ou à l'école, et puis nos aînés restent seuls à la maison...”
Les entrevues nous ont permis d'identifier deux catégories de besoins à caractère socio-affectifs liés à la nécessité d'identification à un groupe pour s'assurer un certain bien-être (affection, amour, acceptation). Il s'agit des besoins socio-culturels et affectifs.

Les besoins socio-culturels sont dominés par le problème de l'isolement individuel et social. L'isolement individuel est caractérisé par l'absence de contact avec les membres de la famille à longueur de jour, alors que l'isolement social a trait à l'absence de contact avec les membres de la communauté d'accueil. Toutes les personnes interviewées ont souligné un désir chez les personnes âgées d'avoir des contacts avec les membres de leurs familles et des opportunités de contacts avec les aînées et aînés de la collectivité d'accueil, dans le but de favoriser des rapprochements, d'avoir des occasions de s'exprimer et de partager les expériences de la vie. Une des représentantes a exprimé le problème d'isolement individuel en ces termes:

Vous savez, nos aînés ont besoin d'un endroit où ils peuvent faire des échanges, socialiser... parce que les familles sont plus ou moins isolées. En Amérique du Nord on est robotisé on peut dire. On se lève le matin, on va travailler ou à l'école, et puis nos aînés restent seuls à la maison...

L'isolement individuel peut être accentué par le fait que lorsque les membres de la famille rentrent du travail ou de l'école, la communication avec les parents âgées n'est pas toujours aisée. À cause, notamment, du stress ou de la fatigue occasionnés par le travail, il est souvent difficile d'être disponible pour répondre aux besoins de communication des parents. Il n'est pas rare que cela engendre des conflits liés aux frustrations accumulées des deux côtés, les parents accusant les enfants de les négliger, et les enfants de leur côté considérant les parents âgés comme un lourd fardeau s'ajoutant aux autres facteurs de stress. On peut aussi comprendre 
"...les personnes âgées

des groupes ethniques

ont aussi plus de

difficultés à améliorer

leurs connaissances de

l'une ou des deux

langues officielles $d u$

pays d'accueil.» que ces réactions soient accentuées par la différence d'obligations face aux personnes âgées ici, par rapport aux pays d'origine. Les enfants peuvent aussi remettre en question les valeurs culturelles du pays d'origine, après avoir été exposés aux valeurs culturelles de la société d'accueil (Nguyen, 1982:32).

Le problème d'isolement est accentué par la température au Canada. Les propos d'une personne âgée interviewée en témoignent: «Vous savez, on vit ici... pour attendre que l'hiver passe vite, je suis vraiment triste de rester seule...» Les facteurs culturels contribuent aussi à l'isolement social chez les personnes âgées des communautés ethniques. La plupart d'entre elles ont un passé culturel qui va dans le sens des interactions, des échanges intenses entre membres de l'environnement. Cela n'étant plus possible dans le pays d'accueil, il devient très difficile de s'adapter à un mode de vie tendant plus vers l'individualisme et la performance personnelle. L'isolement individuel et social est intensifié par le fait que les personnes âgées des groupes ethniques ont aussi plus de difficultés à améliorer leurs connaissances de l'une ou des deux langues officielles du pays d'accueil. Une des représentantes a dit: «La langue est toujours difficile. Il y a beaucoup de problèmes d'analphabétisme dans ma communauté, et il est difficile de trouver des intervenantes ou intervenants qui parlent notre langue». Cette difficulté existe même si la personne parle le français comme langue seconde; elle peut être liée au fait que les personnes ont tendance à perdre leur langue seconde au fur et à mesure qu'elles avancent en âge, ainsi qu'aux incompréhensions résultant des intonations du langage. De plus, la plupart des femmes provenant des pays du Tiers-Monde n'ont pas pu fréquenter l'école, puisqu'elles devaient s'occuper exclusivement des travaux ménagers et de l'éducation des enfants. Les langues des colonisateurs étant surtout utilisées à l'école ou dans les milieux de travail, les personnes qui n'ont pas fréquenté ces milieux ont moins de chance de les connaitre, et de se sentir à l'aise une fois arrivées au Canada. Les possibilités de s'exprimer et de se faire comprendre, lors des contacts sociaux ordinaires avec les agents des institutions offrant des services, sont donc limitées. Il devient aussi difficile d'avoir accès à l'information sur les ressources disponibles et sur les codes 
Reflets

"...dans la plupart

des pays d'origine des

personnes âgées...

celles-ci incarnent la

sagesse accumulée à

travers les expériences

de la vie..» de fonctionnement de la société d'accueil. Dans ces conditions, ces personnes sont plus susceptibles de rester plus dépendantes de leurs enfants, avec toutes les conséquences que cela suppose.

Les programmes de langue seconde ne sont pas nécessairement utiles aux personnes âgées, à cause de l'hétérogénéité des besoins des participantes et participants. Les représentantes rencontrées ont été unanimes pour dire que les personnes âgées n'apprennent pas ces langues au même rythme que d'autres immigrantes ou immigrants adultes, du fait de leur âge.

Je pense qu'on peut donner des cours à nos personnes âgées, qui ne sont pas très avancés pour commencer...

Parce qu'on trouve qu'il y a beaucoup de programmes

d'enseignement de l'anglais et du français comme langues secondes, mais pas pour les personnes âgées. Il serait mieux qu'il $y$ ait un endroit où on peut apprendre les mots d'usage courant pour faciliter l'adaptation, car les personnes âgées ont des difficultés d'apprendre la langue...

Au niveau des besoins affectifs, la moitié des représentantes et la plupart des personnes âgées ont signalé que les personnes âgées ressentent un besoin d'être acceptées et reconnues par les membres de la communauté d'accueil. Ce besoin pourrait découler de leur perception d'un manque d'ouverture pour les contacts sociaux manifesté à leur endroit par le public canadien (Labelle, 1991; Legault, 1991; Bertot et Jacob, 1991). Les possibilités réduites de tissage de nouvelles relations sociales augmentent l'isolement social. La même proportion de répondantes a évoqué les besoins d'estime par les membres de leur famille, de soutien affectif, d'intimité et d'autonomie. Le besoin d'être estimé est étroitement lié au fait que dans la plupart des pays d'origine des personnes âgées considérées, celles-ci incarnent la sagesse accumulée à travers les expériences de la vie. Elles deviennent ainsi vénérées et respectées, ce qui leur confere un pouvoir reconnu par tous les membres de leur famille s'étendant même au niveau des membres de leur entourage global (Minois, 1987). Une fois arrivés au Canada, la plupart de ces aînées et ainnés perdent cette estime, et le choc 
culturel engendré par la différence entre la place accordée aux personnes âgées dans leurs pays d'origine, et celle qui leur est accordée au Canada, les affecte considérablement.

Le besoin d'intimité et d'autonomie est ressenti de façon plus aiguë par les personnes âgées qui sont parrainées par leurs enfants mariés, et qui vivent sous un même toit avec leur beau-fils ou belle-fille. Une représentante disait en effet, que:

...de par les traditions culturelles de son pays d'origine, il est honteux ou scandaleux de voir par exemple son gendre sortant de la salle des bains habillé en peignoir, en pyjama, ou avec un essuie-corps laissant une partie du corps découverte. Vous savez, nos aînés sont comme un oiseau dans une cage une fois qu'ils arrivent ici, et qu'ils sont obligés de vivre avec leurs beauxenfants dans une même maison, souvent exiguë.

On peut supposer que certaines personnes âgées dans cette situation puissent rester presque tout le temps enfermées dans leurs chambres lorsque leur gendre ou leur bru sont à la maison, pour éviter de vivre ce qu'elles considèrent comme un scandale. Nous présumons que le partage de logement avec un parent âgé peut aussi engendrer la même gêne du côté des enfants et contribuer à la diminution de l'intimité conjugale. Pour remédier à cette situation, les personnes âgées devraient avoir leur propre logement, ce qui n'est pas toujours évident à cause, entre autres, de la pénurie et du coût élevé des logements.

Quant au besoin d'autonomie, la moitié des représentantes ont "...les personnes âgées souligné que les personnes âgées des groupes ethniques se plaignent de devoir dépendre de leurs enfants lorsqu'elles ont des besoins financiers. Ces personnes auraient aimé pouvoir subvenir à leurs propres besoins, sans toujours dépendre de la bonne volonté de leurs enfants; quelques personnes âgées interviewées sont également du même avis. Elles se sentent diminuées et dévalorisées dans leur rôle de parents qui doivent, selon leur vision des choses, s'attacher à aider leurs enfants. Une des représentantes interviewées a fait la même observation: 
Reflets

Dans nos pays d'origine, les parents ont le grand plaisir d'aider leurs enfants dans la vie de tous les jours. Vous savez, la plupart des parents âgées ont non seulement la sagesse qui découle de leurs expériences de la vie en société, mais ils ont aussi accumulé des biens matériels qui leur permettent de vivre aisément, en partageant avec leurs enfants et petits-enfants... Il devient difficile de vivre comme avant ici, puisque tous ces biens restent au pays. Vous comprenez que cela cause des frustrations parce qu'ils sont obligés de dépendre uniquement de leurs enfants pour satisfaire à leurs différents besoins.

Ce passage de l'autonomie à la dépendance peut aussi générer une confusion identitaire accompagnée de sentiments de marginalisation et d'aliénation (Berry et al., 1987:492). Certaines personnes âgées ont exprimé le besoin d'encouragement et de soutien de la part des membres de la collectivité d'accueil, lorsqu'elles affrontent les défis reliés au vieillissement et à la vie quotidienne. Une d'entre elles a dit: «La communauté d'accueil devrait aider les personnes âgées, car c'est les derniers jours de la vie. Ça pourrait aider à oublier tout ce que nous avons laissé pour venir au Canada». Cet extrait reflète comment les personnes âgées peuvent se sentir laissées pour compte par la famille et la société. Elles expriment le souhait de réciprocité qui reconnaîtrait que les personnes âgées ont rendu beaucoup de services à la société et que cette dernière doit à son tour leur venir en aide pour la dernière tranche de leur vie (Melchers, 1992).

\section{Ressources formelles et informelles utilisées}

À la lumière des informations recueillies, il appert que les personnes âgées ciblées pour la recherche-intervention ont recours soit aux ressources informelles, soit aux ressources formelles, selon la catégorie de besoins à satisfaire. Mais quelque soit la nature du 
besoin, elles semblent s'adresser surtout aux membres de leur famille. Cette ressource informelle est la plus utilisée par les personnes âgées des groupes ethniques parrainées par leurs enfants, qui en plus d'ignorer d'autres ressources, sont obligées par les politiques sociales, de se tourner vers leurs enfants pour satisfaire la plupart sinon la totalité de leurs besoins pendant une période de dix ans.

Lorsqu'il s'agit de répondre aux besoins à caractère social ou affectif, la majorité de ces personnes âgées se tournent d'abord vers la famille, quitte à approcher par la suite leurs amis ou voisins et enfin les ressources de leurs communautés comme les églises et les associations communautaires. Plusieurs facteurs tels que la culture, le manque d'informations, les difficultés financières et les barrières d'ordre géographique, influencent l'utilisation prononcée des ressources familiales et sociales par rapport aux ressources publiques et communautaires. Au niveau culturel, certaines personnes âgées peuvent éviter d'utiliser les services, soit à cause de la connotation négative reliée à la demande des services aux organismes, soit à cause d'une réticence à partager leurs problèmes d'ordre affectif avec les personnes en dehors de leurs communautés immédiates (McNeely et Colen, 1983:86). Une des représentantes interrogées l'a expliqué ainsi :

Dans notre communauté, les besoins affectifs sont satisfaits par la famille et les contacts sociaux avec l'entourage direct, à travers nos valeurs religieuses où nous nous entraidons tous... Les gens ne s'adressent jamais aux étrangers pour ce genre de besoin.

Les deux catégories de répondantes et répondants s'accordent pour dire que les personnes âgées des groupes ethniques francophones utilisent les ressources formelles pour des problèmes de santé, et dans certains cas pour des besoins financiers. Pour les besoins de santé, elles consultent dans un premier temps les médecins de familles issus de leurs communautés, ce qui leur permet d'éviter les difficultés de communication. Les personnes n'ayant pas accès à cette ressource doivent se faire accompagner par des personnes qui jouent le rôle d'interprètes linguistiques et culturels, afin d'éviter 
Reflets

"...la complexité des démarches à effectuer pour accéder aux ressources formelles fait que certaines personnes âgées se découragent et arrêtent les démarches.» des incompréhensions. Il n'est pas toujours aisé de trouver des accompagnatrices ou accompagnateurs qui soient disponibles au moment où ces personnes âgées en ont besoin.

En ce qui concerne les besoins financiers, celles ou ceux qui remplissent les conditions de d'admission ont recours aux organismes gouvernementaux pour la pension de la vieillesse et les prestations familiales. Plusieurs répondantes et répondants ont souligné que la complexité des démarches à effectuer pour accéder aux ressources formelles fait que certaines personnes âgées se découragent et arrêtent les démarches. Une représentante l'a exprimé en ces termes: «Pour percer cette société d'accueil et bénéficier des services, ça prend des démarches, et puis, ce n'est pas facile, nos aînés ont des difficultés à s'y retrouver». La même observation a été faite par Stevens (1993:285) qui stipule que le système nord-américain sophistiqué est en soi un problème. Les services de la société d'accueil sont très fragmentés, et sous la responsabilité de divers paliers de gouvernements. Cela cause des confusions énormes aux personnes âgées immigrantes qui ont eu l'habitude de recevoir des services dans un même organisme et par les mêmes intervenantes ou intervenants.

Au niveau de la satisfaction des besoins, la plupart des personnes ont parlé des besoins importants qui sont à moitié satisfaits alors que d'autres demeurent non comblés. Il semble que les personnes âgées des groupes ethniques francophones se contentent de ce qu'elles peuvent avoir comme services de la part des membres de leurs familles, même lorsqu'ils ne répondent pas à leurs besoins. Celles qui utilisent les ressources formelles pour combler certains besoins expriment également une sorte d'insatisfaction et d'insécurité. Une personne âgée a dit:

Nous recevons des prestations familiales pour vivre, mais nous avons peur, nous sommes préoccupés parce qu'on envisage couper les prestations familiales. Vous savez, nous avons pris des habitudes de vie avec ce que nous recevons. C'est des revenus modestes, ce n'est pas beaucoup, mais nous pouvons vivre avec ça. Si on coupe, ça va être très difficile... 
"Je suis là, $j$ 'attends toujours les fins de semaine pour que mes enfants s'occupent de moi».
L'insécurité peut être liée au fait que dans la plupart des pays d'origine, on peut se nourrir des produits de la ferme personnelle ou familiale, tout comme on peut combler certains besoins sociaux sans devoir débourser financièrement. Ces ressources n'étant pas disponibles dans la société d'accueil, les aînées et aînés deviennent insécurisés lorsqu'on parle de coupures au niveau des allocations familiales. En ce qui a trait aux besoins socio-culturels tels que les contacts avec la famille, l'entourage direct ou la communauté d'accueil, les ressources utilisées ne semblent pas satisfaisantes. Comme l'illustre cet extrait d'entrevue, les personnes considérées sont très isolées: "J'ai surtout besoin de sortir car rester à la maison, c'est triste. Je pourrais louer des vidéos pour m'occuper si j'avais de l'argent. Je suis là, j'attends toujours les fins de semaine pour que mes enfants s'occupent de moi».

D'autres personnes âgées acceptent simplement le peu de services qu'elles reçoivent, ignorant qu'il existe d'autres possibilités (Legault et Fortin 1993; Duval 1991). Certaines, tout en étant conscientes de l'insuffisance des services offerts par les ressources qu'elles utilisent, se résignent, se sentant impuissantes à changer le fonctionnement de la société d'accueil. Par exemple, la rareté des ressources allouées à l'embauche d'un nombre suffisant d'intervenantes ou intervenants pouvant offrir des services au sein des associations communautaires ethno-culturelles a comme effet de réduire leur fréquence, et ces personnes pensent qu'elles ne peuvent rien y changer.

Il est aussi intéressant de noter que selon les capacités personnelles, certaines personnes âgées tentent des solutions alternatives pour la satisfaction de leurs besoins. C'est le cas d'une personne âgée qui, pour sortir de son isolement individuel et social, se dirige vers les centres d'achat, en vue de pouvoir y rencontrer d'autres personnes de son âge et parler avec elles. 
Reflets

\section{Comment rendre les services francophones plus attirants, accessibles et pertinents?}

"...il incombe aux responsables politiques de créer des opportunités d'apprentissage de ces langues, adaptées aux besoins des personnes âgées.»
Rendre les services de soutien communautaires francophones plus attirants, accessibles et pertinents aux besoins des personnes âgées des groupes ethniques n'est pas facile, si on tient compte de la conjoncture socio-économico-politique canadienne actuelle. Toutefois, nos données sur les obstacles d'accès aux services nous incitent à réfléchir aux stratégies qui pourraient permettre de mieux rejoindre ces personnes. Les obstacles les plus importants identifiés à travers les entrevues sont d'ordre socio-culturel, économique et systémique.

Par rapport aux barrières socio-culturelles, notamment celles à caractère linguistique qui demeurent un obstacle majeur, la facilitation de l'apprentissage ou l'amélioration de la connaissance des langues officielles du Canada pourrait ouvrir la voie à certaines pistes de solution. Pour ce faire, il incombe aux responsables politiques de créer des opportunités d'apprentissage de ces langues, adaptées aux besoins des personnes âgées. Notons que même si les aînées et aînés sont à l'aise en français, l'incompatibilité culturelle des services par rapport aux besoins réels constitue un obstacle qui entrave l'utilisation des services de soutien communautaires. Une répondante a fait la suggestion suivante :

D'abord, il faudrait que dans les activités, on essaie d'introduire celles qui leur sont familières... il serait bon que dans une telle démarche, on cherche à connaître les diverses activités socio-culturelles des différents groupes sociaux... Alors cela serait multicolore et intéresserait tout un chacun. Personne ne se sentirait à l'écart, tout le monde participerait.

L'organisation de programmes sensibles aux valeurs culturelles des participantes et participants immigrants pourrait être une stratégie pour encourager ces derniers à utiliser davantage les organismes 
de soutien communautaires. Comme l'ont écrit Legault et Lafrenière (1991), les services doivent être non seulement accessibles dans un sens géographique, mais aussi au niveau culturel. Les services sociaux et de santé devraient tenir compte des besoins des communautés ethniques afin que ces services puissent refléter davantage les réalités ethno-culturelles du Canada (Auger 1993:164).

Nous avons constaté que les barrières concrètes et systémiques s'influencent mutuellement. La majorité des personnes interrogées ont fait état de difficultés financières. Ce facteur est d'autant plus important compte tenu du fait que dans la société canadienne, ne pas disposer de revenus suffisants implique l'absence de possibilités de choix et d'autonomie (Neysmith, 1985: 208). Le manque de moyens de transport constitue un autre obstacle étroitement lié aux problèmes d'ordre financier. Les politiques sociales, les multiples discriminations caractérisant entre autres le marché du travail canadien constituent des barrières importantes auxquelles les personnes âgées se heurtent quand il s'agit de satisfaire leurs besoins essentiels. En plus de vivre la discrimination linguistique, elles peuvent aussi être victimes de leur âge, de discriminations raciale ou sexuelle dans le cadre de leur recherche de travail. Remédier à la situation de précarité financière à l'origine des multiples difficultés vécues est une tâche de grande envergure puisqu'elle doit comporter des modifications aux politiques d'insertion des immigrantes et immigrants et des réfugiés, qui toucheraient également le marché de l'emploi canadien et les politiques sociales qui y sont liées.

"...certaines mesures concrètes de flexibilité peuvent aider à contourner certains obstacles liés à la précarité financière...»

Bien que la situation s'avère difficile à améliorer, nous estimons que certaines mesures concrètes de flexibilité peuvent aider à contourner certains obstacles liés à la précarité financière, tout en contribuant à gagner la confiance des personnes âgées issues des communautés ethno-culturelles. À titre d'exemple, lorsque nous organisions une activité socio-culturelle pour les aînées et aînés des communautés ethniques francophones, nous nous sommes rendu compte qu'il était difficile de regrouper les personnes âgées des diverses communautés ethno-culturelles francophones, à cause des barrières d'ordre linguistique et culturel. 
Reflets

"...la flexibilité, l'ouverture et la sensibilité face aux valeurs culturelles des personnes âgées des communautés ethniques constituent des éléments importants de succès dans l'organisation d'un programme.»
Nous avons alors opté pour une activité qui a réuni les aînées et aînés d'une des communautés ciblées. Nous avons tenté de lever certaines barrières à la participation, en remboursant entre autres les billets d'autobus, et en offrant un dîner gratuit aux personnes invitées. Cela a permis de recruter un nombre important de personnes qui sont venues participer, et qui ont apprécié l'initiative, en exprimant le souhait que ce genre de rencontres se poursuive. Ces résultats sont venus corroborer ceux d'une expérience similaire à la nôtre, effectuée avec un groupe de personnes âgées d'origine vietnamienne àVancouver. Les organisatrices de l'activité ont conclu que la flexibilité, l'ouverture et la sensibilité face aux valeurs culturelles des personnes âgées des communautés ethniques constituent des éléments importants de succès dans l'organisation d'un programme. (McNicoll et Christensen, 1993).

Nos données révèlent que la quasi totalité des personnes âgées des groupes ethniques francophones ne sont pas suffisamment renseignées au sujet des ressources disponibles dans la communauté, à cause des barrières linguistiques et des approches utilisées pour diffuser l'information. Une personne interrogée a indiqué: «...il faut absolument promouvoir les programmes en les faisant connaitre au sein des communautés ethniques». Ainsi, nous estimons que les organismes de soutien communautaires devraient déployer des efforts pour trouver des moyens plus adaptés et plus créatifs de faire connaitre les services qu'ils offrent. Rejoindre ces personnes âgées n'est certes pas chose facile; cependant, en ayant recours à des personnes relais, aux représentantes ou représentants des communautés ethno-culturelles, on pourrait rejoindre un plus grand nombre de personnes ciblées. Une stratégie culturellement compatible serait l'utilisation de l'approche «bouche à oreille» au sein des différents organismes de soutien communautaires, des paroisses, des hôpitaux, qui pourrait s'ajouter à la diffusion d'informations par les médias de communication communautaires et à l'organisation de forums ou de rencontres d'informations.

Stevens (1993:286) suggérait que la réussite des programmes pour augmenter l'utilisation des services par les nouveaux Canadiens et Canadiennes dépend surtout de la sélection du personnel par 
les agences. Ces derniers devraient obligatoirement comprendre "La modification de la composition $d u$ personnel des organismes pourrait en plus réduire les barrières culturelles liées à la gêne et à la peur de consulter des personnes dans les services formels.» les personnes issues des communautés ethno-culturelles. La modification de la composition du personnel des organismes pourrait en plus réduire les barrières culturelles liées à la gêne et à la peur de consulter des personnes dans les services formels. L'embauche de personnes issues des communautés ethno-culturelles et s'exprimant dans des langues des divers pays d'origine pourrait aussi faciliter la communication verbale et culturelle. Ces personnes serviraient d'intermédiaire ou de référence pour les personnes âgées, comme l'indique la réflexion suivante:

... il faudrait que les divers organismes aient un modèle des ethnies francophones qui partagent la région de la MROC. Afin que lorsque nos aînés ont un besoin à divers niveaux, ils n'éprouvent aucune gêne à s'adresser à tel ou tel autre organisme, compte tenu du fait qu'ils y ont des représentants, des modèles qui peuvent les accueillir, les comprendre et les représenter avantageusement.

La présence d'intervenantes ou d'intervenants issus des communautés ethno-culturelles contribuerait aussi à briser les stéréotypes entretenus par un bon nombre d'immigrantes et immigrants à l'endroit du public canadien, considéré comme raciste. La fréquentation accrue des organismes de soutien communautaires par les personnes âgées des groupes ethniques francophones permettrait un rapprochement avec les personnes âgées canadiennes de souche, maximisant les possibilités de se connaitre, d'échanger des expériences de vie et finalement de mieux se reconnaitre et s'accepter mutuellement. De plus, on pourrait impliquer ces personnes dans les programmes de sensibilisation des intervenantes et intervenants de souche, de façon à augmenter leur compréhension des personnes âgées immigrantes et éventuellement de développer des interventions plus adaptées. 


\section{Conclusion}

Les résultats de notre recherche-intervention démontrent que les personnes âgées des groupes ethniques ont des besoins multiples, touchant plusieurs éléments de la vie en société. Ces personnes utilisent effectivement moins les services de soutien communautaires francophones, même si les ressources vers lesquelles elles se tournent ne répondent pas de façon suffisante à leurs divers besoins. Tout en étant interreliés, leurs multiples besoins sont principalement de deux ordres, à savoir les besoins à caractère tangible et ceux d'ordre socio-affectif. La plupart de ces besoins découlent de leur situation socio-économique et politique au sein de la collectivité d'accueil. Les résultats suggèrent en effet qu'un nombre important de personnes âgées des communautés ethniques vivent dans la précarité financière, ce qui limite considérablement le choix des services pouvant satisfaire à leurs besoins. Afin d'améliorer cette situation, des changements profonds s'imposent au niveau des politiques d'insertion des immigrantes et immigrants en général, et des politiques sociales qui ont trait à la sécurité sociale des personnes âgées immigrantes.

Entre autres, les divers organismes et communautés ethniques concernés par la situation des personnes âgées devraient faire valoir que les dix années d'attente pour bénéficier de la pension de vieillesse sont trop longues pour ces personnes (compte tenu de leur âge), qui ne peuvent même pas travailler au Canada pour avoir un peu de revenu. Cette démarche essentielle contribuerait à ce que les personnes âgées puissent vivre les derniers étapes de la vie avec dignité et de façon épanouissante. De plus, les organismes de soutien communautaires francophones devraient prendre des initiatives pour encourager et faciliter l'accès à leurs services par le biais de tarifs spéciaux, par exemple, ou le remboursement des frais de transport. La mise sur pied d'activités socio-culturelles pour les personnes âgées francophones de souche et celles issues des communautés ethniques favoriserait aussi un rapprochement, facilitant la réduction de l'isolement et l'éclatement de stéréotypes reliés au peu de contacts entre ces groupes. 
Pour faciliter l'apprentissage du français, nous suggérons l'utilisation de l'approche d'alphabétisation-conscientisation développée par Freire, qui consiste à relever l'univers vocabulaire des groupes avec lesquels on travaille (Freire, 1983:259). Notons aussi que la mise en place de lieux d'apprentissage des langues fournirait des occasions permettant aux personnes âgées de socialiser entre elles. Une meilleure maitrise du français favoriserait de plus un rapprochement des diverses communautés, quitte à lever progressivement la peur des incompréhensions mutuelles occasionnée par la rencontre des cultures.

Enfin, soulignons que les personnes âgées des groupes ethniques francophones apportent des expériences de vie qui peuvent enrichir la vie culturelle des francophones au Canada. Si elles restent isolées, elles ne peuvent pas retirer les bénéfices d'un réseau social et communautaire au sein de leur communauté d'accueil. De la même manière, les francophones de souche ne peuvent pas profiter de la richesse de leurs expériences diverses.

Note

Nous remercions l'Association canadienne de gérontologie pour son aide financière qui a facilité notre recherche-intervention.

\section{Bibliographie}

ABOU, Selim (1988). "L'insertion des immigrés: approche conceptuelle», dans P. J. Simon et I. Simon-Barouh, Les étrangers dans la ville, le regard des sciences sociales, Paris, L'Harmattan, pp.1-16.

AUGER, Jeannette A. (1993). «Ethnic Seniors: Accessing Health Services», dans Masi Ralph et al., éd. Health and Cultures: Exploring the Relationships, Programs, Services and Care, Oakville, Mosaic Press, pp.155-167.

BAUKJE, Miedema et Nancy MASON-CLARK (1989). «Second Class Status: An Analysis of the Lived Experience of Immigrant Women in Fredericton", Canadian Ethnic Studies, vol. 21, no 2, pp.63-73.

BEAULIEU, Nadeau et al. (1994). Intégration des communautés ethno-culturelles, Ottawa.

BERRY, J.W. et al. (1987). "Comparative Studies of Acculturative Stress», International Migration Review, vol. 21, no 3, pp.419-511. 
BERGIN, Betty (1988). Une question d'égalité: étude sur l'accès des minorités ethniques aux services de santé et aux services sociaux à Ottawa-Carleton, Ottawa.

BERTOT, Jocelyne et André JACOB (1991). Intervenir avec les immigrants et les réfugiés, Montréal, Méridien Vision.

BIBEAU, G. et al. (1992). La santé mentale et ses visages: un Québec pluriethnique au quotidien, Québec, Gaëtan Morin.

BIBEAU, G. et al. (1987). À la fois d'ici et d'ailleurs: les communautés culturelles du Québec dans les rapports aux services sociaux et aux services de santé (document préparé dans le cadre du programme de recherche de la Commission Rochon).

BOYD, M. (1987). Les immigrantes au Canada: profils et politiques, Ottawa, Emploi et Immigration Canada.

BRANCH, L. G. (1978). Boston Elders (Program report), Boston.

BRETON, Margot. (1991). Ouvrir la pratique du service social de groupe sur la communauté: vers un modèle de partenariat (conférence), Montréal, Université de Montréal.

COCKERHAM, William C. (1991). This Aging Society, Englewood Cliffs/New Jersey, Prentice-Hall.

CONSEIL SUR LEVIEILLISSEMENT (1994). Recueil statistique sur le vieillissement à Ottawa-Carleton, Ottawa.

DEMERS, Sylvie (1987). Émigration, famille, travail et communauté: rôles cachés des femmes portugaises d'Ottawa-Hull, thèse de maîtrise en sociologie, Ottawa, Université d'Ottawa.

DENZIN, N.K. (1987). The Research Act, New York, McGraw-Hill.

DESLAURIERS, Jean-Pierre (1991). Recherche qualitative: Guide pratique, Montréal, McGraw-Hill.

DISMAN, Milada (1987). "Explorations in Ethnic Identity, Oldness, and Continuity", dans C. Gelfand et M. Barresi, ed. Ethnic Dimensions of Aging, New York, Springer, pp.64-78.

DUVAL, Michelle (1991). «Être mère au foyer et récemment immigrée à Montréal: quelques résultats d'une étude exploratoire sur la situation des femmes d'origine vietnamienne, haïtienne et salvadorienne», dans Gilles Pronovost, dir. Comprendre la famille: actes du symposium québécois de recherche sur la famille, Québec, Presses de l'Université du Québec, pp.145-161.

EYRE, Lauren (1992). «Service social multiculturel et personnes âgées», Bulletin du Chapitre de l'Est, vol. 22, no 1.

FREIRE, Paolo (1983). «Suivi de conscientisation et révolution», Pédagogie des opprimés, Paris, Découverte, Maspéro.

GELFAND, Donald E. et Charles M. BARRESI (1987). Ethnic Dimensions of Aging, Springer Series on Adulthood and Aging, New York.

GRINNEL, Richard M. (1988). Social Work Research and Evaluation, 2nd Edition, Itasca, vol. 1, no 11.

GUTTEMANN, D. (1979). "Use of Informal and Formal Supports by the Ethnic Aged», dans D. Gelsand et Kutzik, ed. Ethnicity and Aging: Theory, Research and Policy, New York, Springer.

HERBERG, D. (1993). Frameworks for Cultural and Racial Diversity, Toronto, Scholastic Press.

HOME, Alice et Lise DARVEAU-FOURNIER (1989). Le soutien social aux familles: une stratégie de réduction du stress - Rapport d'un projet pilote, Québec, Université Laval.

LABELLE, Micheline (1990). «Femmes et migration au Canada: bilan et perspectives», Études ethniques au Canada, vol. XXII, no 1, pp.67-82.

LABELLE, Micheline (1987). Histoires d'immigrantes, Montréal, Éditions du Boréal Express. 
LAFOREST, Jacques (1989). Introduction à la gérontologie: croissance et déclin, Montréal, Hurtubise, $\mathrm{HMH}$.

LEGAULT, Giselle et S. FORTIN (1994). «Adaptation-intégration des jeunes familles immigrantes: devis de recherche et problèmes méthodologiques», Intervention, vol. 99, pp.49-63.

LEGAULT, Giselle et Myriam LAFRENI RE (1992). Femmes, migrations, interventions: une rencontre interculturelle - Rapport de recherche, Montréal, École de service social, Université de Montréal.

MAYER, Robert et Francine OUELLET (1991). Méthodologie de recherche pour les intervenants sociaux, Boucherville, Éditions Gaëtan Morin.

McDONALD et WANNER (1990). Retirement in Canada, Butterworths perspectives on individual and adults, Population Aging Series, Toronto/Vancouver.

McNEELY, R. L. et John L. COLEN (1983). Aging in Minority Groups, Beverly Hills, California, Sage Publications.

McNICOLL P. et C. CHRISTENSEN (1993). Making Changes and Making Sense: Social Work Group With Vietnamese Older People, Vancouver, University of British Columbia.

MELCHERS, Ronald (1992). Analyse comparative des services sociaux, cours de maitrise en service social, Ottawa, Université d'Ottawa.

MILES, M. et Michael HUBERMAN (1991). Analyse des données qualitatives: recueil de nouvelles méthodes, Bruxelles, Éditions de renouveau pédagogie.

MINOIS, Georges (1987). Histoire de la vieillesse en Occident: de l'Antiquité à la Renaissance, Paris, Fayard.

NAIDOO, Joséphine et Gary R. EDWARDS (1991). "Combatting Racism Involving Visible Minorities: A Review of Relevant Research and Policy Development», Revue canadienne de service social, vol.VIII, no 2, pp.211-236.

NEYSIMITH, M. Sheila (1985). "Social Work Services to Elder People», dans S. Yelaja, ed. An Introduction to Social Work Practice, Toronto, Prentice-Hall, pp.202-216.

NGUYEN, S. D. (1982). «The Psycho-Social Adjustement and the Mental Needs of Southeast Asian Refugees», The Psychiatric Journal of the University of Ottawa, vol. 7, no 1, pp.26-35.

PATTON, M.Q. (1982). Qualitative Evaluation Methods, Beverly Hills, Sage Publications.

RACINE, Jean-Luc (1994). Nombre de personnes âgées de 65 ans et plus ayant le français comme langue parlée, doc. mineo., Ottawa.

STEVENS, Sarah (1993). «Newcomer Canadians and Mainstream Services», dans Masi Ralph et al., ed. Health and Cultures: Exploring the Relationships, Programs, Services and Care, Canada, Mosaic Press, pp.277-291.

UJIMOTO, Victor K. (1987). «Organizational Activities, Cultural Factors, and Well-Being of Aged Japanese Canadians", dans D. Gelfand et M. Barresi, ed. Ethnic Dimensions of Aging, New York, Springer, pp.145-160. 